\section{Análise espacial dos riscos à \\ saúde associados à incineração \\ de resíduos sólidos: avaliação \\ preliminar}

\author{
Spatial analysis of the health \\ risks associated with solid waste \\ incineration: a preliminary analysis
}

\section{Nelson Gouveia'}

\section{Rogério Ruscitto do Prado}

' Departamento de Medicina Preventiva da Faculdade de Medicina da

Universidade de São Paulo

\section{Resumo}

Objetivo: examinar se as emissões do incinerador de resíduos sólidos Vergueiro estavam associadas a um risco aumentado de câncer entre a população residente em seu entorno. Método: a área de influência deste incinerador foi delimitada por um raio de $7 \mathrm{~km}$ a partir de seu centróide georeferenciado. Os óbitos de indivíduos residentes em distritos administrativos contidos nessa área, no período de 1998 a 2002, por câncer de pulmão, fígado, laringe e linfoma não-Hodgkin em adultos, e por leucemia e todos cânceres combinados em crianças, foram selecionados e geocodificados. A área estudada foi dividida em 7 (sete) anéis concêntricos delimitados por raios de 1 a $7 \mathrm{~km}$ a partir do incinerador. A análise da associação entre proximidade residencial ao incinerador e mortalidade por câncer foi baseada na comparação entre número de casos observados e esperados, utilizando-se o teste de Stone para examinar o declínio do risco (razão O/E) com a distância do incinerador. Resultados: a área estudada incluiu 1.599.532 habitantes, sendo 92.894 crianças $<5$ anos e 634.993 adultos $\geq 40$ anos. Não se observou um gradiente espacial nas razões de mortalidade conforme aumenta a distância do incinerador para nenhuma das causas e morte examinadas. Conclusão: embora não tenha sido detectado aumento no risco dos cânceres previamente selecionados, é importante monitorar as emissões de incineradores ainda em funcionamento e seus possíveis efeitos na saúde. $O$ estudo da distribuição da morbimortalidade em áreas circunvizinhas a essas instalações pode vir a ser uma opção metodológica para atividades de vigilância.

Palavras-chave: Incinerador. Câncer. Mortalidade. Teste de Stone. Análise espacial. 


\section{Abstract}

Objectives: to examine if emissions from the Vergueiro solid waste incinerator are associated with an increased risk of cancer in the population in its vicinity. Methods: the area under influence of this incinerator was delimited by a $7 \mathrm{~km}$ radius from its geocoded centroid. Deaths of city residents in administrative districts inside this area due to cancer of lung, liver, larynx, nonHodgkin's lymphoma in adults, leukemia, and all sites combined in children, in the 1998 to 2002 period, were selected and geocoded. The studied area was divided into 7 concentric rings delimited by a radius of 1 to $7 \mathrm{~km}$ from the incinerator. The analysis of the relationship between residential proximity to the incinerator and mortality due to cancer was based on the comparison of observed and expected cases, using the Stone test for decline in risk with distance from the incinerator. Results: the area studied comprised 1,599,532 inhabitants, of which 92,894 were children less than 5 years old and 634,993 were adults over 40 years old. No spatial gradient in risk was observed for any outcome in relation to distance from the incinerator. Conclusion: although no excess risk for the selected cancers were observed, emissions of incinerators still operating and their possible health effects should be monitored. The study of the spatial distribution of health events in areas around point sources of air pollution can become a methodological option for surveillance activities.

Keywords: Incinerator. Cancer. Mortality. Stone test. Spatial analysis.

\section{Introdução}

A incineração é um método amplamente utilizado na destinação final dos resíduos sólidos em áreas urbanas. Este processo apresenta a vantagem de diminuir o peso e o volume dos resíduos, consequentemente diminuindo os custos de logística e gerenciamento do lixo, além de prevenir o crescimento de bactérias patogênicas e a proliferação de vetores de doenças, usualmente presentes em resíduos orgânicos. Por outro lado, a incineração produz quantidades variadas de substâncias tóxicas, orgânicas ou inorgânicas, que são emitidas na atmosfera.

Partículas, gases, metais pesados, compostos orgânicos, dioxinas e furanos são exemplos de substâncias perigosas emitidas pelos incineradores de resíduos sólidos ${ }^{1-3}$. Muitas dessas substâncias são consideradas potencialmente carcinogênicas para a saúde humana pela International Agency for Research in Cancer (IARC) $)^{4}$. Portanto, suspeita-se que populações residentes em áreas próximas a incineradores estão sujeitas a um risco aumentado de câncer, já que estão expostas a estes componentes diretamente (pela inalação de ar contaminado) ou indiretamente (por meio do consumo de água ou alimentos contaminados, ou contato dérmico com solo contaminado) ${ }^{5}$.

Vários estudos apontam que a exposição da população às emissões de incineradores, na maioria das vezes estimada utilizando alguma medida de distância do incinerador, está associada a um risco aumentado de alguns tipos de câncer, especialmente linfoma não-Hodgkin, sarcomas, câncer de pulmão, fígado e laringe ${ }^{5-12}$. Além disso, alguns estudos têm também relacionado essa exposição a diversos desfechos indesejados da gravidez, incluindo baixo peso ao nascer e anomalias congênitas ${ }^{5,13,14}$.

No entanto, enquanto alguns estudos sugerem algum tipo de efeito na saúde humana, outros estudos são inconclusivos ou não evidenciam qualquer associação $0^{2,15-17}$. Assim, tem sido difícil estabelecer a ocorrência e a magnitude real dos riscos da 
incineração de lixo, informação importante na avaliação das diferentes opções de gerenciamento dos resíduos sólidos.

O Município de São Paulo, com aproximadamente 11 milhões de habitantes, é o maior gerador de resíduos domésticos do país, produzindo diariamente 12.500 toneladas de lixo ${ }^{18}$. A cidade já possuiu quatro grandes incineradores de resíduos sólidos que foram progressivamente desativados com o passar dos anos. O último incinerador em operação foi o Vergueiro, que funcionou entre 1968 e 2002, localizado em uma área com alta densidade populacional. Com uma capacidade nominal de 300 ton/dia, evidenciou-se que as emissões deste incinerador continham concentrações relevantes de arsênico, cádmio e dioxinas ${ }^{19}$. Esse incinerador teve suas operações encerradas devido à persistente mobilização da população local, especialmente após a publicação, no ano 2000, de um relatório técnico indicando contaminação bacteriológica do ar na região do seu entorno (Roberta Simeoni, comunicação pessoal).

Um estudo publicado aproximadamente no mesmo período encontrou uma correlação espacial positiva entre as emissões deste incinerador e eventos mutagênicos medidos em um bioensaio ${ }^{20}$. No entanto, até o momento não foram realizados estudos avaliando o impacto destas emissões na saúde da população residente em sua vizinhança. Desse modo, a partir de registros de mortalidade e utilizando uma abordagem analítica espacial, a presente investigação teve por objetivo avaliar se as emissões deste incinerador estavam associadas a um risco aumentado de câncer entre a população residente em seu entorno.

\section{Métodos}

O incinerador Vergueiro está situado na região sudeste da cidade de São Paulo, em uma área densamente povoada (cerca de $\left.11.000 \mathrm{hab} / \mathrm{km}^{2}\right)^{20}$. Definiu-se a área de influência deste incinerador com base na literatura, com especial atenção ao estudo que mostrou uma queda na frequência de mutações em um bioensaio, de acordo com a distância do incinerador ${ }^{21}$. Portanto, a área de estudo foi delimitada em um raio de 7 $\mathrm{km}$ a partir do centróide georeferenciado do incinerador.

Foram selecionados dados de mortalidade no período de 1998 a 2002, a partir da base de dados do Programa de Aperfeiçoamento das Informações de Mortalidade (PROAIM) do Município de São Paulo. Foram extraídos os óbitos dos sujeitos cuja residência estivesse em um dos distritos administrativos contidos, total ou parcialmente, na área de estudo. Foram selecionados óbitos para as seguintes causas e grupos etários: câncer de pulmão (CID-10 C34.0 - C34.9), câncer de fígado (CID-10 C22.0 - C22.9), câncer de laringe (CID-10 C32.0), linfoma não-Hodgkin (CID-10 C82.0 - C82.9, C83.0 - C83.9, C85.0 - C85.9), para sujeitos $\geq 40$ anos; e todos os cânceres (CID-10 C00 - D48) e leucemia (CID-10 C91 - C95) para crianças < 5 anos. Todas as mortes foram geocodificadas pela variável endereço de residência, utilizando o programa de Sistema de Informação Geográfica (SIG) MapInfo (Professional version 8.5; MapInfo Corporation, New York, NY, USA). Foram também obtidos dados demográficos, como o número de habitantes por idade e gênero, do Censo 2000 para cada setor censitário na área de interesse.

A área estudada foi dividida em 7 anéis concêntricos (Figura 1) delimitados por raios de 1 a $7 \mathrm{Km}$ a partir do incinerador. Cada um desses anéis incluiu todos os setores censitários cujo centróide estivesse contido no anel. Os óbitos foram também designados a cada um desses anéis, de acordo com a distância de seus endereços residenciais em relação ao incinerador. O grupo referência compreendeu aqueles localizados em um raio até $1 \mathrm{~km}$ do incinerador, enquanto os grupos de comparação estavam nos anéis de 1-2 km, 2-3 km e assim por diante.

A análise estatística da associação entre a proximidade residencial ao incinerador e a mortalidade por câncer foi baseada na comparação entre número de casos observados e esperados. Calculou-se o número 


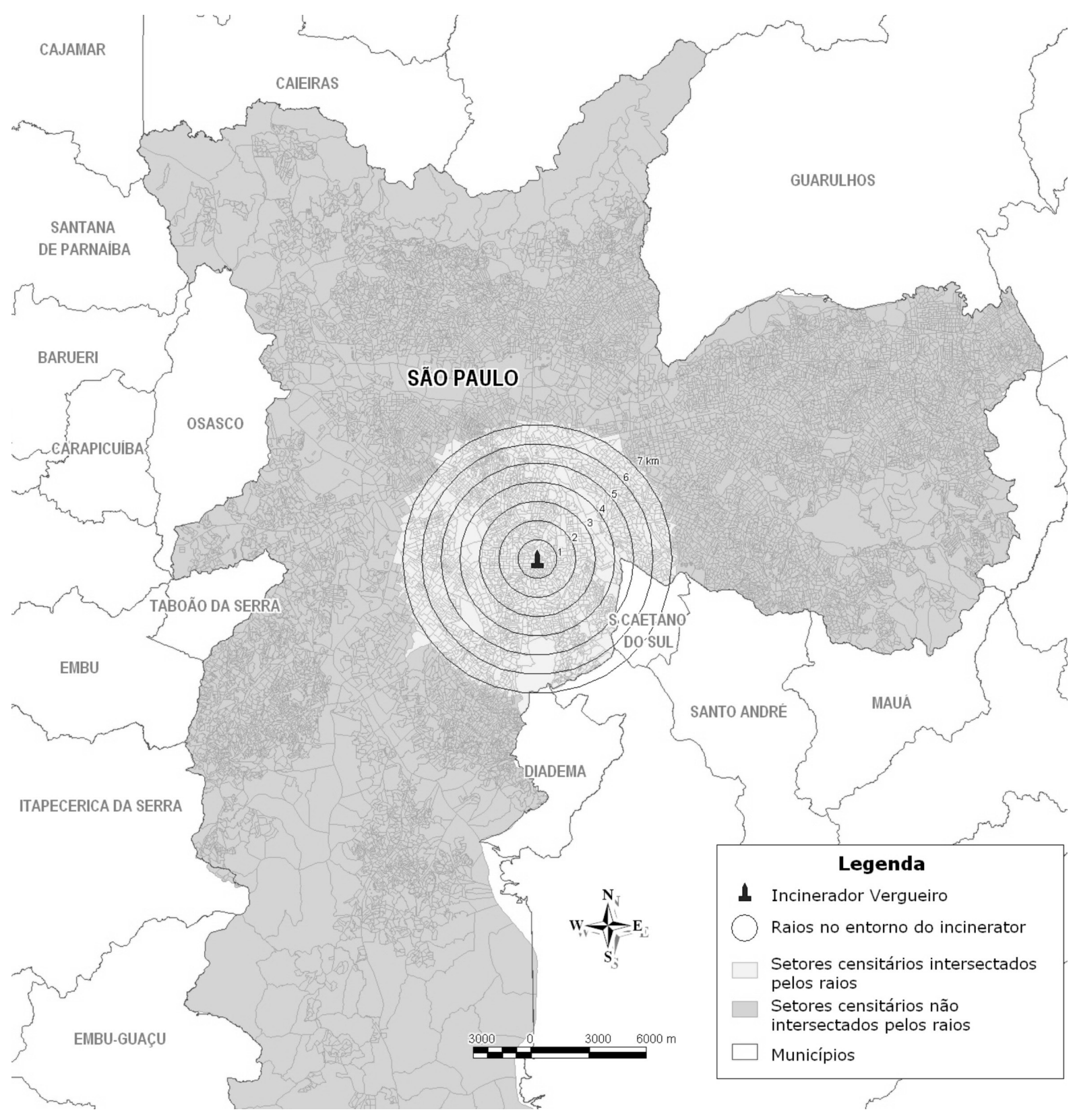

Figura 1 - Mapa do município de São Paulo indicando a localização do Incinerador Vergueiro e as áreas concêntricas em seu entorno.

Figure 1 - Map of the municipality of São Paulo indicating the location of the Vergueiro Incinerator and the concentric buffers around it.

esperado de óbitos em cada anel aplicando as taxas de mortalidade do município para os desfechos estudados, na população por sexo e faixa etária em cada área. Para esses cálculos considerou-se o ano 2000 como referência.

Utilizou-se o teste de Stone ${ }^{22}$ para examinar o declínio do risco (razão O/E) com relação à distância do incinerador. Tomando como base os valores esperados de óbitos para cada área e desfecho, calculados para obter as razões de mortalidade padronizadas (RMP), nessa análise são gerados novos valores esperados supondo uma distribuição multinomial dos óbitos nas várias faixas de distância. Compara-se, então, o número 
de óbitos esperados estimados pelos dois métodos e verifica-se a hipótese (nula) de que não há tendência de decréscimo na RMP conforme se afasta do incinerador.

Este estudo foi aprovado pela Comissão de Ética para Análise de Projetos de Pesquisa - CAPPesq da Diretoria Clínica do Hospital das Clínicas da Faculdade de Medicina da Universidade de São Paulo, sob número 1009/02.

\section{Resultados}

A área de até $7 \mathrm{~km}$ a partir do incinerador incluiu 26 dos 96 distritos administrativos do município de São Paulo. Nessa área, distribuídos em 120.038 domicílios, reside uma população de 1.599 .532 habitantes, sendo 92.894 crianças $<5$ anos e 634.993 pessoas $\geq 40$ anos. A distribuição dessa população entre as áreas ao redor do incinerador Vergueiro estão detalhadas na Tabela 1 .

As causas de morte selecionadas para este estudo totalizaram 3.456 óbitos nos 26 distritos. Desses, apenas 14 (0.4\%) não puderam ser georeferenciados, pela ausência do endereço residencial (10 eventos) ou devido a endereço incorreto (4 eventos). Assim, 3.442 óbitos foram incluídos no estudo, mas, desses, apenas 2.924 (85\%) estavam dentro do raio de até $7 \mathrm{~km}$ a partir do incinerador. A grande maioria desses óbitos (61,9\%) foi por câncer de pulmão, seguido de câncer de fígado (15,6\%) e linfoma nãoHodgkin (12,8\%). Os óbitos de menores de 5 anos por todos os cânceres representaram apenas 1,3\% do total de casos selecionados nesse estudo.

O número de óbitos observado e o esperado em cada anel, para cada uma das causas selecionadas, assim como as razões de mortalidade padronizadas, estão dispostos na Tabela 2. O teste de Stone para declínio nos riscos não revelou gradiente espacial nas razões de mortalidade, segundo a distância do incinerador, para nenhuma das causas examinadas. Nota-se que o número de óbitos por câncer em crianças é bastante baixo em cada área estudada, o que dificulta uma avaliação mais precisa nesse grupo etário.

\section{Discussão}

Embora o incinerador Vergueiro tenha sido objeto de algumas investigações e tenha sido identificado que emitia substâncias potencialmente tóxicas à saúde humana ${ }^{19}$, este é o primeiro estudo a avaliar o impacto dessas emissões diretamente na saúde da população residente em sua vizinhança. Entretanto, os resultados do presente estudo não corroboram a hipótese de que residência em áreas próximas a esse incinerador aumenta o risco de alguns tipos de câncer. Não foram encontrados indícios

Tabela 1 - População e domicílios, por anéis concêntricos no entorno do Incinerador Vergueiro, e no município de São Paulo, 2000.

Table 1 - Population and households in the concentric buffers bordering the Vergueiro Incinerator and in the municipality of São Paulo, 2000.

\begin{tabular}{lcccc}
\hline Anéis $(\mathrm{Km})$ & Domicílios & $<5$ anos & $\geq 40$ anos & Total \\
\hline 0 a 1 & 13.658 & 2.311 & 16.975 & 42.638 \\
1 a 2 & 35.615 & 5.350 & 46.308 & 108.217 \\
2 a 3 & 66.989 & 11.500 & 84.049 & 204.738 \\
3 a 4 & 77.269 & 15.788 & 90.966 & 243.836 \\
4 a 5 & 98.293 & 16.487 & 118.073 & 292.445 \\
5 a 6 & 121.478 & 19.671 & 141.662 & 352.018 \\
6 a 7 & 120.038 & 21.787 & 136.960 & 355.640 \\
\hline Total da área & 533.340 & 92.894 & 634.993 & 1.599 .532 \\
\hline São Paulo & 2.998 .445 & 879.083 & 3.176 .313 & 10.434 .252 \\
\hline
\end{tabular}


Tabela 2 - Mortes observadas e esperadas nos anéis no entorno do IRS Vergueiro, por causas específicas, São Paulo, 1998-2002

Table 2 - Observed and expected deaths in the households in the concentric buffers bordering the Vergueiro Incinerator, by specific causes, São Paulo, 1998-2002

\begin{tabular}{|c|c|c|c|c|c|c|c|c|c|}
\hline & \multirow[t]{2}{*}{ Desfecho } & \multicolumn{8}{|c|}{ Anéis (Km) } \\
\hline & & 0 a 1 & 1 a 2 & 2 a 3 & 3 a 4 & 4 a 5 & 5 a 6 & 6 a 7 & p Stone \\
\hline \multirow{16}{*}{$\begin{array}{l}\text { o } \\
\frac{C}{N} \\
\stackrel{+}{+} \\
\text { NI }\end{array}$} & Fígado & & & & & & & & 0,672 \\
\hline & Observado & 9 & 37 & 61 & 64 & 97 & 92 & 95 & \\
\hline & Esperado & 9 & 25 & 45 & 48 & 63 & 75 & 73 & \\
\hline & RMP & 1,00 & 1,50 & 1,36 & 1,32 & 1,54 & 1,22 & 1,30 & \\
\hline & Laringe & & & & & & & & 0,418 \\
\hline & Observado & 5 & 22 & 29 & 26 & 45 & 52 & 56 & \\
\hline & Esperado & 6 & 17 & 32 & 34 & 44 & 53 & 52 & \\
\hline & RMP & 0,78 & 1,26 & 0,92 & 0,76 & 1,01 & 0,98 & 1,09 & \\
\hline & Linfoma & & & & & & & & 0,420 \\
\hline & Observado & 11 & 31 & 35 & 57 & 67 & 80 & 92 & \\
\hline & Esperado & 7 & 20 & 36 & 39 & 50 & 60 & 58 & \\
\hline & $\underline{\mathrm{RMP}}$ & 1,53 & 1,58 & 0,98 & 1,48 & 1,34 & 1,33 & 1,59 & \\
\hline & Pulmão & & & & & & & & 0,354 \\
\hline & Observado & 43 & 151 & 231 & 257 & 304 & 424 & 400 & \\
\hline & Esperado & 35 & 97 & 176 & 190 & 247 & 296 & 286 & \\
\hline & RMP & 1,21 & 1,56 & 1,31 & 1,35 & 1,23 & 1,43 & 1,40 & \\
\hline \multirow{8}{*}{ 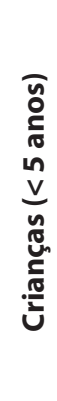 } & Todos os cânceres & & & & & & & & 0,835 \\
\hline & Observado & 0 & 2 & 6 & 7 & 3 & 10 & 11 & \\
\hline & Esperado & 1 & 2 & 4 & 5 & 5 & 6 & 7 & \\
\hline & RMP & 0,00 & 1,13 & 1,58 & 1,34 & 0,55 & 1,54 & 1,53 & \\
\hline & Leucemia & & & & & & & & 0,556 \\
\hline & Observado & 0 & 1 & 2 & 2 & 0 & 6 & 1 & \\
\hline & Esperado & 0 & 0 & 1 & 1 & 1 & 2 & 2 & \\
\hline & RMP & 0,00 & 2,35 & 2,18 & 1,59 & 0,00 & 3,83 & 0,58 & \\
\hline
\end{tabular}

de associação entre proximidade residencial ao incinerador Vergueiro e cânceres de fígado, laringe, e linfoma não-Hodgkin, para sujeitos $\geq 40$ anos; e todos os cânceres ou leucemia para crianças $<5$ anos.

Há grande controvérsia na literatura sobre a existência de efeitos deletérios à saúde associados às emissões dos incineradores. Elliott et al. ${ }^{9}$ examinaram a incidência de câncer em pessoas residentes entre 3 e 7,5 km de incineradores de resíduos sólidos no Reino Unido e encontraram um declínio estatisticamente significante no risco para todos os cânceres. Em um estudo na França, por meio de uma análise espacial sofisticada, Viel et al. ${ }^{10}$ encontraram aglomerações (clusters) estatisticamente significantes para sarcoma de tecidos moles e linfoma não-Hodgkin em áreas no entorno de um incinerador. Em Roma, Michelozzi et al. ${ }^{6}$ encontraram um declínio estatisticamente significante na mortalidade por câncer de laringe entre homens conforme aumentava a distância de diferentes fontes de poluição atmosférica, incluindo um incinerador. Por outro lado, muitos estudos não encontraram qualquer associação com câncer ou outros desfechos ${ }^{2,15-17}$. 
Uma das limitações importantes do presente estudo foi a utilização de dados de mortalidade por câncer obtidos das declarações de óbito (DO). Apesar da alta qualidade dos diagnósticos, uma vez que a grande maioria das DO em São Paulo é preenchida por médicos, e frequentemente o PROAIM realiza verificações nos procedimentos para garantir a qualidade dos registros, a mortalidade por câncer é um evento extremo e mais relacionado ao acesso adequado a diagnóstico e tratamento. Entretanto, inexistem bases de dados completas e fidedignas sobre a incidência de câncer para o Município de São Paulo para utilização em estudos epidemiológicos.

Além disso, o câncer é uma doença que tem um período de indução bastante extenso. Também não foi possível considerar a quantidade de tempo realmente despendida por cada sujeito naquele endereço. Neste estudo, a exposição de cada indivíduo foi definida de acordo com seu local de residência no momento da morte. Essas limitações são relativamente menos importantes quando se avalia a mortalidade por câncer em crianças menores de 5 anos, uma vez que o tempo de exposição e de indução para o surgimento do câncer fica delimitado a, no máximo, 5 anos. Todavia, o número de eventos para esse grupo etário foi pequeno, não permitindo uma avaliação mais rigorosa. Desfechos relacionados à gravidez, como o baixo peso ao nascer ou as malformações congênitas, podem ser indicadores muito mais sensíveis em avaliações futuras dessa exposição.

Outra limitação relevante em estudos desse tipo é que importantes variáveis de confusão, tais como tabagismo, exposições ocupacionais e condição socioeconômica, tampouco puderam ser consideradas nessas análises.

Esse estudo assumiu que a exposição foi homogênea dentro de cada raio ao redor do incinerador. Essa suposição não leva em conta o regime de ventos nem as condições climáticas que podem influenciar a dispersão dos poluentes emitidos. Embora seja razoável supor que as condições de operação do incinerador foram regulares, não havendo grandes variações temporais nos níveis de emissão, a utilização de um modelo de dispersão Gaussiano refletiria muito melhor a exposição da população nas vizinhanças.

Os mecanismos pelos quais os poluentes emitidos afetam a saúde humana são variados e dependem do tipo de substância predominantemente liberada. Algumas das que são reconhecidamente emitidas por incineradores têm algum potencial carcinogênico já comprovado ${ }^{4}$. Efeitos mutagênicos em vegetais já foram observados nas áreas sujeitas às emissões do incinerador Vergueiro $^{20}$.

Embora não tenha sido detectado nenhum aumento no risco dos cânceres previamente selecionados para análise nesse estudo, a preocupação com as emissões de incineradores de resíduos sólidos deve ser mantida. A incineração é uma das opções para o gerenciamento de resíduos em áreas urbanas, um problema cada vez maior para as cidades. A proporção do lixo produzido que tem sido destinada à incineração vem crescendo em muitos países ${ }^{3}$. Mesmo que os equipamentos hoje utilizados disponham de novas tecnologias, é necessário manter um monitoramento de suas emissões e de seus possíveis efeitos na saúde.

Uma das possibilidades metodológicas para o monitoramento de efeitos é o estudo da distribuição da morbimortalidade por determinadas causas em áreas circunvizinhas a essas instalações. Com as bases de dados contendo informações sociodemográficas e de saúde hoje disponíveis, a avaliação do impacto à saúde das emissões de fontes fixas por meio de análise espacial pode revelar-se uma ferramenta útil para atividades de vigilância. Esse tipo de investigação, por ser relativamente rápido de ser executado e a custos baixos, pode ser a primeira abordagem na identificação de riscos à saúde da população. Assim, medidas preventivas podem ser tomadas rapidamente, enquanto se espera pelo resultado de estudos específicos e mais detalhados. 


\section{Referências}

1. Rushton L. Health hazards and waste management. $\mathrm{Br}$ Med Bull 2003; 68: 183-97.

2. Vinceti M, Malagoli C, Teggi S, Fabbi S, Goldoni C, De Girolamo G, et al. Adverse pregnancy outcomes in a population exposed to the emissions of a municipal waste incinerator. Sci Total Environ 2008; 15; 407(1): 116-21.

3. World Health Organization. Population health and waste management: scientific data and policy options. Report of a WHO workshop Rome, Italy, 29-30 March 2007. Copenhagen: WHO Regional Office for Europe; 2007.

4. International Agency for Research in Cancer. IARC Monographs on the Evaluation of Carcinogenic Risks to Humans: Polychlorinated Dibenzo-para-dioxins and Polychlorinated Dibenzofurans, vol. 69. Lion: IARC; 1997.

5. Franchini M, Rial M, Buiatti E, Bianchi F. Health effects of exposure to waste incinerator emissions: a review of epidemiological studies. Ann 1st Super Sanità. 2004; 40(1): 101-15.

6. Michelozzi P, Fusco D, Forastiere F, Ancona C, Dell'Orco V, Perucci CA. Small area study of mortality among people living near multiple sources of air pollution. Occup Environ Med 1998; 55(9): 611-5.

7. Biggeri A, Barbone F, Lagazio C, Bovenzi M, Stanta G. Air Pollution and Lung Cancer in Trieste, Italy: Spatial Analysis of Risk as a Function of Distance from Sources. Environ Health Persp 1996; 104(7): 750-4.

8. Watanabe S, Kitamura K, Nagahashi M. Effects of dioxins on human health: a review. J Epidemiol 1999; 9(1): 1-13.

9. Elliott P, Shaddick G, Kleinschmidt I, Jolley D, Walls P, Beresford J et al. Cancer incidence near municipal solid waste incinerators in Great Britain. Br J Cancer 1996; 73: 702-10.

10. Viel JF, Arveux P, Baverel J, Cahn JY. Soft-Tissue Sarcoma and Non-Hodkin's Lymphoma Clusters around a Municipal Solid Waste Incinerator with high dioxin emission levels. Am J Epidemol 2000; 152(1):13-9.

11. Zambon P, Ricci P, Bovo E, Casula A, Gattolin M, Fiore $\mathrm{AR}$, et al. Sarcoma risk and dioxin emissions from incinerators and industrial plants: a population-based case-control study (Italy). Environ Health 2007; 16: 6-19.

12. Viel JF, Daniau C, Goria S, Fabre P, Crouy-Chanel P, Sauleau EA, et al. Risk for non Hodgkin's lymphoma in the vicinity of French municipal solid waste incinerators. Environ Health 2008; 7: 51-9.
13. Cordier S, Chevrier C, Robert-Gnansia E, Lorente C, Brula P, Hours M. Risk of congenital anomalies in the vicinity of municipal solid waste incinerators. Occup Environ Med 2004; 61(1): 8-15.

14. Tango T, Fujita T, Tanihata T, Minowa M, Doi Y, Kato N, et al. Risk of adverse reproductive outcomes associated with proximity to municipal solid waste incinerators with high dioxin emission levels in Japan. J Epidemiol 2004; 14(3): 83-93.

15. Elliott P, Hills M, Beresford J, Kleinschmidt I, Jolley D, Pattenden S et al. Incidence of cancers of the Larynx and lung near incinerators of waste solvents and oils in Great Britain. Lancet 1992; 339: 854-8.

16. Viel JF, Clement MC, Hägi M, Grandjean S, Challier B, Danzon A. Dioxin emissions from a municipal solid waste incinerator and risk of invasive breast cancer: a population-based case-control study with GIS-derived exposure. Int J Health Geogr 2008; 7: 4-11.

17. Vinceti M, Malagoli C, Fabbi S, Teggi S, Rodolfi R, Garavelli L, et al. Risk of congenital anomalies around a municipal solid waste incinerator: a GIS-based casecontrol study. Int J Health Geogr 2009; 10(8): 8-17.

18. Cetesb. Inventário estadual de resíduos sólidos domiciliares: Relatório de 2008. Cetesb; 2009.

19. Fruehauf GLC. A deterioração da qualidade do ar e do clima na região metropolitana de São Paulo. [tese de doutorado]. São Paulo: FFLCH; 1998.

20. SEADE. Município de São Paulo - MSP. São Paulo; 2008. Disponível em http://seade.sp.gov. br/produtos/msp/ index.php. [Acessado em junho de 2009.]

21. Ferreira MI, Petrenko H, Lobo DJ, Rodrigues GS, Moreira A, Saldiva PH. In situ monitoring of the mutagenic effects of the gaseous emissions of a solid waste incinerator in metropolitan Sao Paulo, Brazil, using the Tradescantia stamen-hair assay. J Air Waste Manag Assoc 2000; 50: 1852-6.

22. Stone R. Investigations of excess environmental risks around putative sources: statistical problems and a proposed test. Statistics in Medicine 1988; 7: 649-60.

Recebido em: 16/09/09

Versão final reapresentada em: 23/02/10

Aprovado em: 24/02/10 